\title{
Theoretical analysis of a nearly optimal analog quantum search
}

\author{
Carlo Cafaro ${ }^{1}$ and Paul M. Alsing ${ }^{2}$ \\ ${ }^{1}$ SUNY Polytechnic Institute, 12203 Albany, New York, USA and \\ ${ }^{2}$ Air Force Research Laboratory, Information Directorate, 13441 Rome, New York, USA
}

\begin{abstract}
We analyze the possibility of modifying the original Farhi-Gutmann Hamiltonian algorithm in order to speed up the procedure for producing a suitably distributed unknown normalized quantum mechanical state. Such a modification is feasible provided only a nearly optimal fidelity is sought. We propose to select the lower bounds of the nearly optimal fidelity values such that their deviations from unit fidelity are less than the minimum error probability characterizing the optimum ambiguous discrimination scheme between the two nonorthogonal quantum states yielding the chosen nearly optimal fidelity values. Departing from the working assumptions of perfect state overlap and uniform distribution of the target state on the unit sphere in $N$-dimensional complex Hilbert space, we determine that the modified algorithm can indeed outperform the original analog counterpart of a quantum search algorithm. This performance enhancement occurs in terms of speed for a convenient choice of both the ratio $\gamma=E^{\prime} / E$ between the energy eigenvalues $E^{\prime}$ and $E$ of the modified search Hamiltonian and the quantum mechanical overlap $x$ between the source and the target states. Finally, we briefly discuss possible analytical improvements of our investigation together with its potential relevance in practical quantum engineering applications.
\end{abstract}

PACS numbers: Quantum computation (03.67.Lx), Quantum information (03.67.Ac). 


\section{INTRODUCTION}

In 1997, Grover presented a quantum algorithm for solving very large database search problems [1], one of the outstanding problems in computational science [2]. Grover's search algorithm enables searching for an unknown marked item in an unstructured database of $N$ items by accessing the database a minimum number of times. From a classical standpoint, one needs to test $N / 2$ items, on average, before identifying the correct item. With Grover's algorithm however, the same task can be completed successfully with a complexity of order $\sqrt{N}$, that is, with a quadratic speed up. The original formulation of Grover's algorithm was presented in terms of a discrete sequence of unitary logic gates (digital quantum computation). Alternatively, Farhi and Gutmann proposed an analog version of Grover's algorithm in Ref. 3] where the state of the quantum register evolves continuously in time under the action of a suitably chosen driving Hamiltonian (analog quantum computation). For recent discussions on the transition from the digital to analog quantum computational setting for Grover's algorithm, we refer to Ref. [4]. The formulation of the analog algorithm proposed by Farhi and Gutmann can be briefly described as follows. Consider $N$ mutually orthonormal states and assume that only one of them has non-zero energy (energetically marked state). Starting from an equal superposition of the $N$ states, the problem is to determine how quickly the system can evolve under a given Hamiltonian so that the energetically marked state is reached with certainty. Within the working assumption of time-independent Hamiltonian evolutions, Farhi and Gutmann showed that their algorithm required a minimum time of the order $\sqrt{N}$, thus yielding the same complexity as Grover's original algorithm. Interestingly, the evolution time for the analog algorithm scaled like $\sqrt{N} / E$, with $E$ denoting the energy of the marked state, just as demanded by the time-energy uncertainty principle [7]. In Refs. [7 9], it was shown that the minimum time interval required for an isolated quantum system to evolve to an orthogonal (that is, perfectly distinguishable) state is inversely proportional to its constant energy spread. Such minimum time is also known as orthogonalization time in the literature. In Ref. [10, the maximum speed of evolution of an isolated quantum system was interpreted in terms of the maximum number of distinct states that the system can explore in a fixed time interval. This fixed time interval was shown to be inversely proportional to the system's quantum mechanical average energy minus its ground state energy. For a unified tight bound on the rate of quantum dynamical evolution based on both the average energy and the energy spread of the system, we refer to Ref. [11]. The presence of a finite non-orthogonality in the context of quantum limits to dynamical evolutions was considered, for instance, in Ref. [12] and Ref. [13]. In the former work, the expression of the states that minimize the time necessary to evolve, under a time-independent Hamiltonian, from an initial quantum state to a final state that is only approximately orthogonal was found. It is worth noting that the 
final state is only approximately orthogonal for a given upper bound on the mean energy of a general closed system. In the latter work, on the contrary, an extension of the concept of quantum speed limit to mixed states in the case where the quantum system does not evolve from an initial state to an orthogonal state (that is, the case of non-zero fidelity) was proposed.

In our paper, instead of allowing for a finite non-orthogonality between the quantum states that leads to a nonzero fidelity, we consider a complementary scenario. We allow for a finite non-parallelism that yields a non-unit fidelity. The consideration of nearly optimal state searching is instructive for various reasons. First, it is reasonable to take into account the fact that actual measurements always involve a countable set of outcomes and thus, any realistic measurement device has finite resolution [14. Second, finite resolution measurements can reveal more about the physical properties of quantum systems than precise measurements [15]. Third, it is known that a quantum measurement can enhance the transition probability between two pure states [16] and, finally, it is indisputable that quantum measurement cannot perfectly discriminate between two nonorthogonal pure states [17].

Motivated by these considerations, the questions we address in this paper can be outlined as follows: First, what is the minimum time necessary to reach a given nearly optimal fidelity value in a continuous time quantum search algorithm? Second, is it possible to formulate a modified version of the original Farhi-Gutmann search algorithm that leads to such a selected fidelity value in a shorter time than the one required by the original Farhi-Gutmann algorithm? Third, how can we reasonably justify the choice of a lower bound for such a nearly optimal fidelity value? We demonstrate that it is possible to extend the original work performed by Farhi and Gutmann in order to speed up the procedure for producing a suitably distributed unknown normalized quantum state with high non-unit fidelity. In particular, by modifying the original working assumptions of perfect state overlap and uniform distribution of the target state on the unit sphere in the $N$-dimensional complex Hilbert space, the newly proposed algorithm can indeed outperform the original analog counterpart of a quantum search algorithm. This is achieved for a convenient choice of both the ratio $\gamma \stackrel{\text { def }}{=} E^{\prime} / E$ between the eigenvalues $E^{\prime}$ and $E$ of the modified search Hamiltonian and the quantum mechanical overlap $x$ between the initial and target states.

The layout of the remainder of this paper is as follows. In Section II, we extend the Farhi-Gutmann optimality proof to the case of an imperfect quantum state overlap. In Section III, we present the transition probability for the modified version of the Farhi-Gutmann analog algorithm. In Section IV, we explain how we choose the lower bounds of the nearly optimal fidelity values by means of quantum discrimination techniques. Specifically, we impose that deviations from unit fidelity are less than the minimum error probability characterizing the optimum ambiguous 
discrimination scheme between the two nonorthogonal quantum states yielding the selected nearly optimal fidelity values. In Section V, we compare in terms of minimum run time the original and the modified quantum search algorithms and identify a two-dimensional parametric region where the modified algorithm outperforms the original one under the working assumption of imperfect state transfer compatible with finite precision quantum measurements. Our concluding remarks appear in Section VI. Finally, technical details on the numerical values of the quantum overlap $x$ can be found in Appendix A.

\section{THE MODIFIED OPTIMALITY PROOF}

In this section, we modify the original optimality proof by Farhi and Gutmann in Ref. [3] by relaxing the requirement of finding the desired target state with certainty. In particular, we wish to determine how the search time shortens if we only want to achieve a nearly optimal fidelity value.

The optimality proof provided by Farhi and Gutmann in Ref. 3] for producing the target state $|w\rangle$ relies on two key working assumptions. First, the target state is produced with absolute certainty. In other words, the modulus squared of the quantum state overlap, that is, the transition probability or transition fidelity at the end of the production procedure is equal to unity. Second, the target state $|w\rangle$ is an unknown element of a given orthonormal basis $\{|a\rangle\}$ with $1 \leq a \leq N$ of an $N$-dimensional complex Hilbert space $\mathcal{H}_{2}^{n}$ with $N \stackrel{\text { def }}{=} 2^{n}$. Our modified optimality proof for an imperfect quantum state overlap follows closely the lines of the original optimality proof presented in Ref. [3]. Consider a quantum system, assumed to be initially in a state $|i\rangle$ at time $t=0$ that does not depend on the target state $|w\rangle$ and evolves by means of a Hamiltonian $\mathcal{H}$ given by,

$$
\mathcal{H} \stackrel{\text { def }}{=} \mathcal{H}_{w}+\mathcal{H}_{D}
$$

where $\mathcal{H}_{w} \stackrel{\text { def }}{=} E|w\rangle\langle w|$. For the discussion that follows, it is unnecessary to specify the explicit expression for the driving Hamiltonian $\mathcal{H}_{D}$ in Eq. (1). The problem being investigated is that of finding a lower bound $\tilde{t}$ on the time $t$ for the $|w\rangle$-independent state $|i\rangle$ to evolve into the state $|w\rangle$ given that its evolution is governed by the time-independent Hamiltonian $\mathcal{H}$ in Eq. (1). Let us consider the following two initial value problems,

$$
\left\{\begin{array} { c } 
{ i \hbar \frac { d } { d t } | \psi _ { w } ( t ) \rangle = \mathcal { H } | \psi _ { w } ( t ) \rangle } \\
{ | \psi _ { w } ( 0 ) \rangle = | i \rangle }
\end{array} , \text { and } \left\{\begin{array}{c}
i \hbar \frac{d}{d t}|\psi(t)\rangle=\mathcal{H}_{D}|\psi(t)\rangle \\
|\psi(0)\rangle=|i\rangle
\end{array}\right.\right.
$$

where $\hbar$ denotes the reduced Planck constant. In what follows, we depart from Farhi and Gutmann original optimality proof. We do not require achieving the absolute certainty of producing the target state at the end of the evolution 
and assume that at time $\tilde{t}$ we have,

$$
\left|\psi_{w}(\tilde{t})\right\rangle \stackrel{\text { def }}{=}\left\langle w \mid \psi_{w}(\tilde{t})\right\rangle|w\rangle+\left\langle w_{\perp} \mid \psi_{w}(\tilde{t})\right\rangle\left|w_{\perp}\right\rangle
$$

The normalization condition for $\left|\psi_{w}(\tilde{t})\right\rangle$ requires that the probability amplitudes satisfy the following constraint equation,

$$
\left|\left\langle w \mid \psi_{w}(\tilde{t})\right\rangle\right|^{2}+\left|\left\langle w_{\perp} \mid \psi_{w}(\tilde{t})\right\rangle\right|^{2}=1
$$

The quantity $\left|w_{\perp}\right\rangle$ denotes a (normalized) state orthogonal to the target state $|w\rangle$ contained in the two-dimensional subspace of the full Hilbert space spanned by $|w\rangle$ and $\left|w_{\perp}\right\rangle$. A suitable choice for the parametrization of the state $\left|\psi_{w}(\tilde{t})\right\rangle$ in Eq. $(3)$ is given by,

$$
\left|\psi_{w}(\tilde{t})\right\rangle=\cos (\delta)|w\rangle+e^{-i\left(\theta_{w}-\theta_{w_{\perp}}\right)} \sin (\delta)\left|w_{\perp}\right\rangle
$$

where $\theta_{w}$ and $\theta_{w_{\perp}}$ are arbitrary real phases while $\delta$ is assumed to be a small and positive real parameter. Finally, for convenience and without loss of generality, we set $\theta_{w}-\theta_{w_{\perp}}=\pi / 2$ so that $\left|\psi_{w}(\tilde{t})\right\rangle$ becomes

$$
\left|\psi_{w}(\tilde{t})\right\rangle=\cos (\delta)|w\rangle-i \sin (\delta)\left|w_{\perp}\right\rangle
$$

where $i$ denotes the complex imaginary unit. In what follows, we assume $0 \leq \delta \ll 1$ so that $\cos ^{2}(\delta)=1-\delta^{2}+\mathcal{O}\left(\delta^{4}\right)$ and $\sin ^{2}(\delta)=\delta^{2}+\mathcal{O}\left(\delta^{4}\right)$. For the sake of clarity, we point out that the validity of this latter working assumption will be explicitly clarified by means of Eq. (38) and Fig. 2 in the next section. Furthermore, we have

$$
\cos (\delta)=1-(1 / 2) \delta^{2}+\mathcal{O}\left(\delta^{4}\right), \text { and } \sin (\delta)=\delta+\mathcal{O}\left(\delta^{3}\right)
$$

To find a lower bound $\tilde{t}$ on the time $t$, we proceed as follows. First, consider the following quantity

$$
\sum_{w=1}^{N} \|\left|\psi_{w}(\tilde{t})\right\rangle-|\psi(\tilde{t})\rangle \|^{2}
$$

By using Eqs. (6) and (7) in the expression for $\|\left|\psi_{w}(\tilde{t})\right\rangle-|\psi(\tilde{t})\rangle \|^{2}$ appearing in Eq. (8), we obtain after some algebra,

$$
\|\left|\psi_{w}(\tilde{t})\right\rangle-|\psi(\tilde{t})\rangle \|^{2}=2-\cos (\delta)[\langle\psi(\tilde{t}) \mid w\rangle+\langle w \mid \psi(\tilde{t})\rangle]+i \sin (\delta)\left[\left\langle\psi(\tilde{t}) \mid w_{\perp}\right\rangle-\left\langle w_{\perp} \mid \psi(\tilde{t})\right\rangle\right]
$$

Substituting Eq. (9) into Eq. (8), we obtain

$$
\left.\sum_{w=1}^{N}\|\| \psi_{w}(\tilde{t})\right\rangle-|\psi(\tilde{t})\rangle \|^{2}=2 N-\cos (\delta) \sum_{w=1}^{N}[\langle\psi(\tilde{t}) \mid w\rangle+\langle w \mid \psi(\tilde{t})\rangle]+i \sin (\delta) \sum_{w=1}^{N}\left[\left\langle\psi(\tilde{t}) \mid w_{\perp}\right\rangle-\left\langle w_{\perp} \mid \psi(\tilde{t})\right\rangle\right]
$$


Observe that for a set of complex amplitude coefficients $\left\{c_{j}\right\}$ with $1 \leq j \leq N$, we find

$$
\sum_{j=1}^{N}\left|c_{j}\right|^{2}=1 \Rightarrow \sum_{j=1}^{N}\left|c_{j}\right| \leq N^{\frac{1}{2}}
$$

Therefore, by exploiting Eq. (11), we observe that

$$
\sum_{w=1}^{N} \|\left|\psi_{w}(\tilde{t})\right\rangle-|\psi(\tilde{t})\rangle \|^{2} \geq 2 N-2 \sqrt{N} \cos (\delta)-2 \sqrt{N} \sin (\delta) .
$$

By using Eq. (7) in Eq. (12) and neglecting second order terms in $\delta$ we arrive at,

$$
\sum_{w=1}^{N} \|\left|\psi_{w}(\tilde{t})\right\rangle-|\psi(\tilde{t})\rangle \|^{2} \geq(2 N-2 \sqrt{N})(1-\delta)+2(N-2 \sqrt{N}) \delta .
$$

Observe that for $N \geq 4$, we have both $2 N-2 \sqrt{N} \geq N$ and $N-2 \sqrt{N} \geq 0$. Finally, Eq. 13 reduces to,

$$
\sum_{w=1}^{N} \|\left|\psi_{w}(\tilde{t})\right\rangle-|\psi(\tilde{t})\rangle \|^{2} \geq N(1-\delta)
$$

Now, let us consider the following expression,

$$
\frac{d}{d t} \|\left|\psi_{w}(t)\right\rangle-|\psi(t)\rangle \|^{2}
$$

After some algebra and exploiting the normalization conditions $\left\langle\psi_{w}(t) \mid \psi_{w}(t)\right\rangle=1$ and $\langle\psi(t) \mid \psi(t)\rangle=1$, we obtain

$$
\|\left|\psi_{w}(t)\right\rangle-|\psi(t)\rangle \|^{2}=2-\left\langle\psi_{w}(t) \mid \psi(t)\right\rangle-\left\langle\psi(t) \mid \psi_{w}(t)\right\rangle .
$$

Differentiating with respect to time the LHS (left-hand-side) and the RHS (right-hand-side) of Eq. (16) and recalling that $z+z^{*}=2 \operatorname{Re}(z)$ for any $z \in \mathbb{C}$ where $z^{*}$ denotes the complex conjugate of $z$, we find

$$
\frac{d}{d t} \|\left|\psi_{w}(t)\right\rangle-|\psi(t)\rangle \|^{2}=-2 \operatorname{Re}\left\{\frac{d}{d t}\left[\left\langle\psi_{w}(t) \mid \psi(t)\right\rangle\right]\right\}
$$

that is,

$$
\frac{d}{d t} \|\left|\psi_{w}(t)\right\rangle-|\psi(t)\rangle \|^{2}=-2 \operatorname{Re}\left[\left\langle\frac{d\left|\psi_{w}(t)\right\rangle}{d t} \mid \psi(t)\right\rangle+\left\langle\psi_{w}(t) \mid \frac{d|\psi(t)\rangle}{d t}\right\rangle\right]
$$

Recalling that $i \hbar \frac{d}{d t}\left|\psi_{w}(t)\right\rangle=\mathcal{H}\left|\psi_{w}(t)\right\rangle$ and $i \hbar \frac{d}{d t}|\psi(t)\rangle=\mathcal{H}_{D}|\psi(t)\rangle$ and observing that $\operatorname{Re}(i z)=-\operatorname{Im}(z)$ for any $z \in \mathbb{C}$, Eq. 18 becomes

$$
\frac{d}{d t} \|\left|\psi_{w}(t)\right\rangle-|\psi(t)\rangle \|^{2}=\frac{2}{\hbar} \operatorname{Im}\left[\left\langle\psi_{w}(t)\left|\mathcal{H}_{w}\right| \psi(t)\right\rangle\right]
$$


Employing the Cauchy-Schwarz inequality and recalling that $\left\langle\psi_{w}(t) \mid \psi_{w}(t)\right\rangle=1$, we obtain

$$
\begin{aligned}
\frac{d}{d t} \|\left|\psi_{w}(t)\right\rangle-|\psi(t)\rangle \|^{2} & =\frac{2}{\hbar} \operatorname{Im}\left[\left\langle\psi_{w}(t)\left|\mathcal{H}_{w}\right| \psi(t)\right\rangle\right] \\
& \leq \frac{2}{\hbar}\left|\left\langle\psi_{w}(t)\left|\mathcal{H}_{w}\right| \psi(t)\right\rangle\right| \\
& \leq \frac{2}{\hbar} \|\left|\psi_{w}(t)\right\rangle\|\cdot\| \mathcal{H}_{w}|\psi(t)\rangle\left\|=\frac{2}{\hbar}\right\| \mathcal{H}_{w}|\psi(t)\rangle \| .
\end{aligned}
$$

Summing over the index $w$ in Eq. 20], we have

$$
\frac{d}{d t}\left(\sum_{w=1}^{N} \|\left|\psi_{w}(t)\right\rangle-|\psi(t)\rangle \|^{2}\right) \leq \frac{2}{\hbar} \sum_{w=1}^{N} \| \mathcal{H}_{w}|\psi(t)\rangle \| .
$$

Since $\mathcal{H}_{w} \stackrel{\text { def }}{=} E|w\rangle\langle w|$ and $\langle w \mid w\rangle=1$, and using Eq. 11, the RHS in the inequality written in Eq. 21, becomes

$$
\frac{2}{\hbar} \sum_{w=1}^{N} \| \mathcal{H}_{w}|\psi(t)\rangle \|=\frac{2 E}{\hbar} \sum_{w=1}^{N}|\langle w \mid \psi(t)\rangle| \leq \frac{2 E}{\hbar} \sqrt{N} .
$$

Combining Eqs. 21) and 22, we obtain

$$
\frac{d}{d t}\left(\sum_{w=1}^{N} \|\left|\psi_{w}(t)\right\rangle-|\psi(t)\rangle \|^{2}\right) \leq \frac{2 E}{\hbar} \sqrt{N} .
$$

Integrating Eq. 23] with respect to time and recalling the initial conditions $\left|\psi_{w}(0)\right\rangle=|\psi(0)\rangle=|i\rangle$, we find

$$
\sum_{w=1}^{N} \|\left|\psi_{w}(t)\right\rangle-|\psi(t)\rangle \|^{2} \leq \frac{2 E}{\hbar} \sqrt{N} t .
$$

Finally, by combining Eq. 14 and Eq. 24 evaluated at $t=\tilde{t}$, we have

$$
N(1-\delta) \leq \sum_{w=1}^{N} \|\left|\psi_{w}(\tilde{t})\right\rangle-|\psi(\tilde{t})\rangle \|^{2} \leq \frac{2 E}{\hbar} \sqrt{N} \tilde{t},
$$

that is,

$$
\frac{2 E}{\hbar} \sqrt{N} \tilde{t} \geq N(1-\delta)
$$

or, equivalently,

$$
\tilde{t} \geq \frac{\hbar}{2 E}(1-\delta) \sqrt{N}
$$

Equation 27) is our first finding reported in this paper and can be regarded as the analog of Eq. (23) in Ref. [12] obtained in the context of finding states that minimize the evolution time to become an almost orthogonal state. 
We point out that although it is expected that $\tilde{t}_{\min }^{\text {(approximate) }} \leq \tilde{t}_{\min }^{\text {(exact) }}$, the scaling behavior of $\tilde{t}_{\text {min }}^{\text {(exact) }}$ as a function of the angle $\delta$ with $0 \leq \delta \ll 1$ is not at all obvious. We emphasize that the validity of Eq. (27) is limited to the case in which the target state $|w\rangle$ is an unknown element of a given orthonormal basis $\{|a\rangle\}$ with $1 \leq a \leq N$ of an $N$-dimensional complex Hilbert space. We also recall that $\delta$ is defined by the relation $\delta \stackrel{\text { def }}{=} \cos ^{-1}\left[\left\langle w \mid \psi_{w}(\tilde{t})\right\rangle\right]$ with $\left|\psi_{w}(t)\right\rangle \stackrel{\text { def }}{=} \hat{U}\left(t, t_{0}\right)\left|\psi_{w}\left(t_{0}\right)\right\rangle$. The unitary operator $\hat{U}\left(t, t_{0}\right) \stackrel{\text { def }}{=} e^{-\frac{i}{\hbar} \mathcal{H}\left(t-t_{0}\right)}$ is the usual quantum mechanical evolution operator. Therefore in general, the quantity $\delta$ can depend on certain characteristics of the chosen time-independent search Hamiltonian $\mathcal{H}$.

For the sake of clarity, we point out that our proposed modified optimality proof is not meant to be used to determine the optimality of a modified continuous time quantum search algorithm. The lower bound in Eq. (27) is only useful for providing the optimality of the scaling of the complexity of any algorithm specified by the Hamiltonian in Eq. (1). In particular, its purpose is to find out the behavior with respect to the parameter $\delta$ of the minimum time necessary to achieve a desired nearly optimal transition probability value (that is, $\cos ^{2}(\delta)$ with $\left.0 \leq \delta \ll 1\right)$ when evolving from a source state $|i\rangle$ to an approximate target state $\left|\psi_{w}(\tilde{t})\right\rangle$ under the Hamiltonian in Eq. (1). In other words, we have not compared the optimality of two distinct algorithms that aim to solve the same identical task. Instead, we are interested in finding out how the performance of a single algorithm, quantified in terms of the minimum time necessary to accomplish the task, is affected when two distinct tasks have to be completed. In our case, the first task (that is, the original task) is to find the target state with certainty (that is, success probability equal to one). The second task (that is, the modified task), instead, is to search for an approximate target state that corresponds to a success probability that is only nearly equal to one. In summary, when considering the original task,

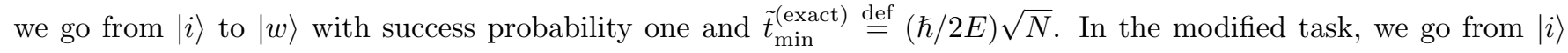

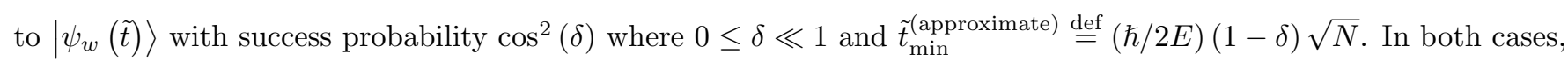
the same algorithm described by the Hamiltonian in Eq. (1) is employed.

Our proposed modified optimality proof allowed us to formally introduce the parameter $\delta$ in a general physical setting where it is not necessary to specify the explicit expression of the driving Hamiltonian $\mathcal{H}_{D}$ in Eq. (1). The following section, instead, includes a more specific discussion on the functional form of the parameter $\delta$ for a specific choice of the driving Hamiltonian. 


\section{THE MODIFIED FARHI-GUTMANN SEARCH HAMILTONIAN}

In this section, we present the transition probability for the modified version of the Farhi-Gutmann analog search algorithm. Assume that the time-independent Hamiltonian $\mathcal{H}$ in Eq. (1) is given by,

$$
\mathcal{H} \stackrel{\text { def }}{=} \mathcal{H}_{w}+\mathcal{H}_{D}=E|w\rangle\left\langle w\left|+E^{\prime}\right| s\right\rangle\langle s|
$$

where,

$$
\mathcal{H}_{w} \stackrel{\text { def }}{=} E|w\rangle\langle w| \text { and, } \mathcal{H}_{D} \stackrel{\text { def }}{=} E^{\prime}|s\rangle\langle s|
$$

Observe that when $E^{\prime}=E$, the Hamiltonian $\mathcal{H}$ reduces to the Hamiltonian as originally proposed by Farhi and Gutmann in Ref. [3]. Furthermore, assume that $|w\rangle$ is the normalized target state while $|s\rangle$ is the normalized initial state with $\langle s \mid w\rangle=x$ that evolves in an unitary fashion according to Schrödinger's quantum mechanical evolution law,

$$
|s\rangle \mapsto e^{-\frac{i}{\hbar} \mathcal{H} t}|s\rangle
$$

where the time-independent Hamiltonian $\mathcal{H}$ is given in Eq. 28. We wish to compute the time $t^{*}$ such that the transition probability $\mathcal{P}(t)$ defined as,

$$
\mathcal{P}(t) \stackrel{\text { def }}{=}\left|\left\langle w\left|e^{-\frac{i}{\hbar} \mathcal{H} t}\right| s\right\rangle\right|^{2}
$$

assumes its maximum value $\mathcal{P}\left(t^{*}\right)=\mathcal{P}_{\max }$. Introducing the orthonormal basis $\{|w\rangle,|r\rangle\}$ with $|r\rangle \stackrel{\text { def }}{=}$ $\left(1-x^{2}\right)^{-\frac{1}{2}}(|s\rangle-x|w\rangle)$ and $|s\rangle \stackrel{\text { def }}{=} x|w\rangle+\sqrt{1-x^{2}}|r\rangle$, after some straightforward but tedious matrix algebra computations, the explicit expression $\mathcal{P}\left(t ; x, E, E^{\prime}\right)$ of $\mathcal{P}(t)$ in Eq. 31 becomes

$$
\mathcal{P}\left(t ; x, E, E^{\prime}\right)=\frac{x^{2}(1+\gamma)^{2}}{4 x^{2} \gamma+(1-\gamma)^{2}} \sin ^{2}\left(\frac{1}{2 \hbar} \sqrt{4 x^{2} E E^{\prime}+\left(E^{\prime}-E\right)^{2}} t\right)+x^{2} \cos ^{2}\left(\frac{1}{2 \hbar} \sqrt{4 x^{2} E E^{\prime}+\left(E^{\prime}-E\right)^{2}} t\right)
$$

where $\gamma \stackrel{\text { def }}{=} E^{\prime} / E$ in Eq. 32 . Observe that for $E=E^{\prime}$, we recover from the expression of $\mathcal{P}\left(t ; x, E, E^{\prime}\right)$ in Eq. 32 the original fidelity expression as obtained by Farhi and Gutmann in Ref. [3],

$$
\mathcal{P}_{\text {Farhi-Gutmann }}(t ; x, E)=\sin ^{2}\left(\frac{E x}{\hbar} t\right)+x^{2} \cos ^{2}\left(\frac{E x}{\hbar} t\right)
$$

To find the instant $t^{*}$ for which $\mathcal{P}\left(t^{*} ; x, E, E^{\prime}\right)=\mathcal{P}_{\max }$, we proceed as follows. First, from Eq. 32, we observe that $\mathcal{P}_{\max }$ equals

$$
\mathcal{P}_{\max }(x, \gamma)=\frac{x^{2}(1+\gamma)^{2}}{4 x^{2} \gamma+(1-\gamma)^{2}}
$$




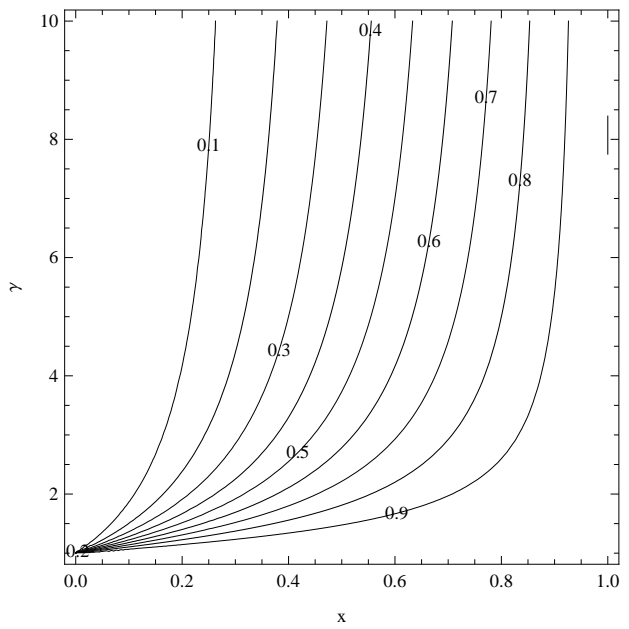

FIG. 1: Contour plot exhibiting $\gamma$ v.s. $x$ with $1 \leq \gamma \leq 10$ and $0 \leq x \leq 1$. The level curves are given by $P_{g}^{\max }(x, \gamma)=c$ where $c$ is a constant such that $0.1 \leq c<1$.

In Fig. 1, we present the contour plot that exhibits $\gamma$ v.s. $x$ with $1 \leq \gamma \leq 10$ and $0 \leq x<1$. In particular, the level curves are defined by the relation $\mathcal{P}_{\max }(x, \gamma)=c\left(\right.$ with $\mathcal{P}_{\max }(x, \gamma)=\mathcal{P}_{\mathrm{g}}^{\max }$ in Eq. 48 ) where $c$ is a constant with numerical values such that $0.1 \leq c<1$. As a side remark, notice that when $E=E^{\prime}$, that is $\gamma=1, \mathcal{P}_{\max }=1$ for any value of $x$ in agreement with what was reported in Ref. 3 . Second, the instant $t^{*}$ for which $\mathcal{P}\left(t^{*} ; x, E, E^{\prime}\right)=\mathcal{P}$ max with $\mathcal{P}\left(t ; x, E, E^{\prime}\right)$ and $\mathcal{P}_{\max }$ in Eqs. 32 and 34 , respectively, satisfies the relation

$$
\frac{1}{2 \hbar} \sqrt{4 x^{2} E E^{\prime}+\left(E^{\prime}-E\right)^{2}} t^{*}=\frac{\pi}{2}
$$

that is, since $E^{\prime}=\gamma E$,

$$
t^{*}(x, \gamma) \stackrel{\text { def }}{=} \frac{\pi \hbar}{2 E} \frac{2}{\sqrt{4 x^{2} \gamma+(1-\gamma)^{2}}} .
$$

Once again, observe that in the limiting case of $\gamma=1$, we recover the original relation found in Ref. [3],

$$
t_{\text {Farhi-Gutmann }}^{*}(x) \stackrel{\text { def }}{=} \frac{\pi \hbar}{2 E} \frac{1}{x}
$$

We recall that at the end of Section II, we stated that the quantity $\delta$ can depend on specific properties of the Hamiltonian $\mathcal{H}$ in Eq. (28). By using Eqs. (3) and (34), we can finally obtain the explicit functional form of $\delta$ in terms of the two parameters $x$ and $\gamma$,

$$
\delta=\delta(x, \gamma) \stackrel{\text { def }}{=} \cos ^{-1}\left[\frac{(1+\gamma) x}{\sqrt{(1-\gamma)^{2}+4 \gamma x^{2}}}\right] .
$$

Using Eq. (38), we present in Fig. 2 a plot of $\delta(x)$ versus $x$ for a number of fixed values of $\gamma$ greater than one. Finally, observe that for $\gamma=1, \delta(x, \gamma)=0$ for any choice of $x$ and we recover the limiting case discussed in Ref. [3]. 


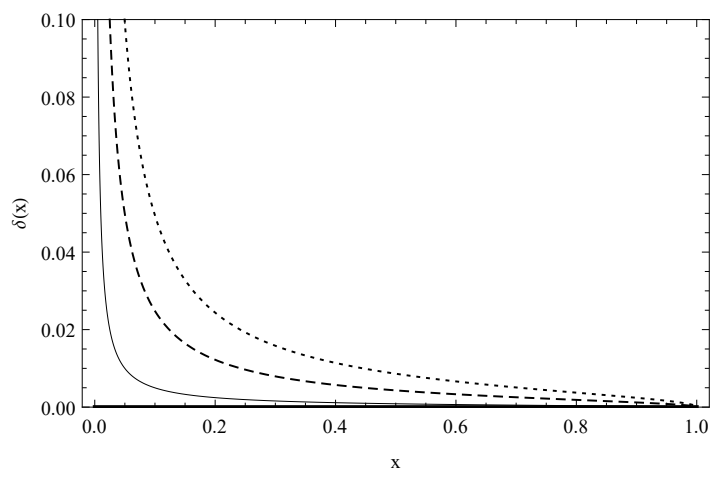

FIG. 2: Plot of $\delta(x)$ v.s. $x$ assuming that $\gamma=1$ (thick line), $\gamma=1.001$ (thin line), $\gamma=1.010$ (dashed line), and $\gamma=1.1$ (dotted line).

\section{LOWER BOUNDS ON THE FIDELITY VALUES}

Having introduced the parameter $\delta$ in a general setting in Section II (see Eq. 27)) and in a more specific framework in Section III (see Eq. (38)), we would like to address in Section IV the following question: How big can $\delta$ be chosen? In principle, we could arbitrarily choose a nearly optimal threshold value of the error probability for quantum search. However, in what follows, we propose a way to justify to a certain extent this arbitrariness with some plausible line of reasoning. First, at the end of the search, we expect that the approximate and the exact target states will be very close. Therefore, the error probability for quantum search (that is, its deviation from the unit fidelity) will be very small. Second, since these target states have a large overlap, it will be difficult to distinguish them. Therefore, we also expect that the error probability for quantum state discrimination will be large. Given these two considerations, it is intuitive that in our working conditions the error probability for quantum search will be smaller than the error probability for quantum state discrimination. However, even in the presence of large overlaps between quantum states, it is possible to device highly successful quantum state discrimination protocols where the minimum error probability can be made arbitrarily small. We propose to select the minimum success probability values for quantum search by imposing that the deviation from the unit fidelity is smaller than the smallest (compatible with realistic finite-precision quantum measurements) minimum error probability achievable in highly successful quantum state discrimination protocols for quantum states with very large overlaps.

If we desire to consider a very small departure $\Delta \mathcal{F}(\delta)$ from the unit fidelity,

$$
\Delta \mathcal{F}(\delta) \stackrel{\text { def }}{=} \mathcal{F}_{\text {optimal }}-\mathcal{F}_{\text {nearly-optimal }}(\delta)=1-\cos ^{2}(\delta),
$$


we expect $0 \leq 1-\cos ^{2}(\delta) \leq \varepsilon$ with $0 \leq \varepsilon \ll 1$, that is

$$
0 \leq \delta \leq \delta_{\max }(\varepsilon) \stackrel{\text { def }}{=} \cos ^{-1}(\sqrt{1-\varepsilon})
$$

Therefore, the question becomes: How small should the real parameter $\varepsilon$ in Eq. 40 be chosen? How do we select this value? Can we physically motivate such a choice for $\varepsilon$ ? In what follows, motivated by theoretical quantum state discrimination principles [17, 18] and acknowledging the presence of imperfections in actual quantum measurement settings that limit the precision of the measurement of angles [19, 20, we propose a way to select the numerical upper (lower) bounds for $\delta\left(\mathcal{F}_{\text {nearly-optimal }}(\delta)\right)$.

We recall that the goal in a quantum state discrimination problem is to identify the actual state of a quantum system that is prepared, with a fixed prior probability, in a specific but unknown state that belongs to a finite set of given possible states [17, 18. An essential property of quantum mechanics is that two pure states cannot be distinguished perfectly, if they are not orthogonal. Therefore, there are fundamental limitations in quantum state discrimination protocols on the success with which one can determine the actual state. Specifically, when the possible states are not mutually orthogonal, it is not possible to develop a state-distinguishing protocol that can discriminate between them perfectly. Relevant state-distinguishing techniques are the unambiguous [21 23] and the ambiguous 24 26] state discrimination schemes. Unambiguous state discrimination requires that, whenever a definite (that is, conclusive) outcome is recorded after the measurement, the result must be error free (that is, unambiguous). When the measurement fails to provide a definite outcome, a nonzero probability of inconclusive outcomes has to be taken into consideration. Then, the optimum unambiguous discrimination scheme is realized when the failure probability (that is, the probability of inconclusive outcomes) is minimum. On the contrary, ambiguous state discrimination requires that a conclusive outcome has to be recorded in each single measurement. This in turn implies that the discrimination is ambiguous since errors in the conclusive result are unavoidable. Based on the measurement outcome, a guess is made as to what the actual state of the quantum system was. Then, the optimum ambiguous discrimination scheme is obtained when the error probability (that is, the probability of making a wrong guess) is minimum. For a very instructive discussion on how to interpret the original Farhi-Gutmann analog quantum search algorithm as a method for improving the distinguishability of a set of Hamiltonians by adding a controlled driving term, we refer to Ref. [27].

Being in the framework of ambiguous quantum state discrimination, assume that we are given one of two normalized quantum states $\left|\psi_{1}\right\rangle=|w\rangle$ and $\left|\psi_{2}\right\rangle=|\tilde{w}\rangle$, not necessarily orthogonal, with prior probabilities $p_{1}=p_{w}$ and $p_{2} \stackrel{\text { def }}{=}$ $1-p_{1}=p_{\tilde{w}}$, respectively. Furthermore, assume we have been asked to optimally determine which state we have 


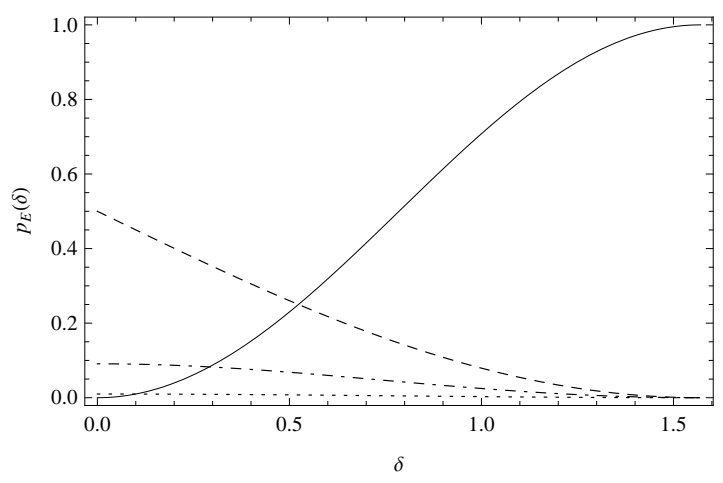

FIG. 3: Plot of the minimum error probability $p_{E}(\delta)$ v.s. the angle $\delta$ assuming that $p_{w}=p_{\tilde{w}}\left(\right.$ dashed line), $p_{w}=10 p_{\tilde{w}}$ (dot-dashed line), and $p_{w}=10^{2} p_{\tilde{w}}$ (dotted line). The thin line denotes the deviation $\Delta \mathcal{F}$ from the optimal fidelity.

actually been given. It happens that the minimum error probability $p_{E}(\delta)$ is given by [17, 18,

$$
p_{E}(\delta)=\frac{1}{2}\left(1-\sqrt{1-4 p_{w} p_{\tilde{w}} \cos ^{2}(\delta)}\right)
$$

From Eq. 41], we note that the minimum error probability $p_{E}$ is a monotonic increasing function of the quantum mechanical overlap $\langle w \mid \tilde{w}\rangle \stackrel{\text { def }}{=} \cos (\delta)$. In particular, when $\delta$ approaches zero (that is, very large quantum overlap) and $p_{w}=p_{\tilde{w}}$, it can become quite challenging to distinguish the state $|\tilde{\omega}\rangle$ from the state $|\omega\rangle$ since the probability of making an incorrect guess approaches 50\%. Alternatively, we point out that in the presence of large asymmetry between the prior probabilities $p_{w}$ and $p_{\tilde{w}}$, the success of the discrimination protocol increases and, as a consequence, it becomes less difficult to discriminate between $|w\rangle$ and $|\tilde{w}\rangle$. For instance, when $p_{\tilde{w}}=10^{-2} p_{w}$, the minimum error probability $p_{E}$ approaches the liming value $10^{-2}$ as the angle $\delta$ approaches zero. We observe that the quantum mechanical overlap $\langle w \mid \tilde{w}\rangle$ plays a key role in both quantum state discrimination and nearly optimal analog quantum search. In the former case, it is the essential quantity that sets a bound to the effectiveness of the discrimination scheme. In the latter case, it quantifies the departure from the perfect unit fidelity. In view of the above mentioned considerations, it becomes useful to define an approximate target state $|\tilde{w}\rangle$ as a quantum state with a nearly optimal fidelity $\mathcal{F}_{\text {nearly-optimal }}(\delta) \stackrel{\text { def }}{=} \cos ^{2}(\delta)$ that departs from the unit perfect fidelity $\mathcal{F}_{\text {optimal }} \stackrel{\text { def }}{=} 1$ by an amount $\Delta \mathcal{F}(\delta) \stackrel{\text { def }}{=} \mathcal{F}_{\text {optimal }}-\mathcal{F}_{\text {nearly-optimal }}(\delta)$. We impose that the quantity $\Delta \mathcal{F}(\delta)$ is smaller than the minimum error probability $p_{E}(\delta)$ in Eq. 41, ,

$$
0 \leq \Delta \mathcal{F}(\delta) \stackrel{\text { def }}{=} \mathcal{F}_{\text {optimal }}-\mathcal{F}_{\text {nearly-optimal }}(\delta) \leq p_{E}(\delta)
$$

Eq. 42 implies that we choose to select the lower bounds of the nearly optimal fidelity values $\left\{\mathcal{F}_{\text {nearly-optimal }}(\delta)\right\}$ in such a manner that their deviations $\{\Delta \mathcal{F}(\delta)\}$ from the unit fidelity $\mathcal{F}_{\text {optimal }}$ are less than the minimum error probability $p_{E}(\delta)$ characterizing the optimum ambiguous discrimination scheme between the two nonorthogonal quantum states 
$\{|\tilde{\omega}\rangle,|\omega\rangle\}$ (that is, the approximate and the exact target states, respectively) yielding the chosen nearly optimal fidelity values. A plot of $p_{E}(\delta)$ as a function of the angle $\delta$ for a variety of sets of a priori probabilities appears in Fig. 3. We point out that the proposed inequality in Eq. $\sqrt{42}$ is reminiscent of the (sandwich) Fuchs-van de Graaf inequalities relating the trace norm distance and the fidelity as suitable measures of closeness for quantum states [28]. Indeed, the trace norm distance appears in the expression for the minimum error probability while the fidelity appears in the equation for the transition probability in our discussion. For the sake of completeness, we also remark that while Fuchs and van de Graaf were only concerned with the problem of distinguishing between two quantum states with equal a priori probabilities, an extension of their analysis to the case of arbitrary a priori probabilities can be found in Ref. [29]. As a final consideration, we emphasize that in the limiting case of equal a priori probabilities together with the conditions $0 \leq \delta \ll 1$, it is straightforward to verify that our inequality in Eq. 42 can be obtained from the Fuchs-van de Graaf inequalities. In particular, as explained below, the sharpness of the bounds that we propose in Eq. (42) can be tuned by means of the degree of asymmetry that specifies the a priori probabilities of the two quantum states being distinguished.

Recalling Eq. (40), the problem was the determination of how small the real parameter $\varepsilon$ in Eq. (40) should be. We are now in the condition to provide an answer to this issue. We note that, after some straightforward algebra and using Eq. (39), the inequality constraint in Eq. (42) yields

$$
0 \leq \delta \leq \delta_{\max }\left(\bar{p}_{w}\right) \stackrel{\text { def }}{=} \cos ^{-1}\left[\sqrt{1-\bar{p}_{w}\left(1-\bar{p}_{w}\right)}\right]
$$

The quantity $\bar{p}_{w}$ in Eq. (43) denotes a fixed value of the prior probability $p_{w}$. Knowing that $\bar{p}_{\tilde{w}}+\bar{p}_{w}=1$ and setting $\bar{p}_{\tilde{w}}=\alpha \bar{p}_{w}$ with $\alpha$ being the degree of asymmetry between the two a priori probabilities $\bar{p}_{\tilde{w}}$ and $\bar{p}_{w}$, Eq. 43) becomes

$$
0 \leq \delta \leq \delta_{\max }(\alpha) \stackrel{\text { def }}{=} \cos ^{-1}\left[\sqrt{1-\alpha(1+\alpha)^{-2}}\right]
$$

We observe that in the maximally symmetric scenario, we have $\left(\bar{p}_{w}, \bar{p}_{\tilde{w}}\right)=(1 / 2,1 / 2)$ (that is, $\left.\alpha=1\right)$. Instead, in the maximally asymmetric scenario, we have $\left(\bar{p}_{w}, \bar{p}_{\tilde{w}}\right)=(1,0)$ (that is, $\left.\alpha \ll 1\right)$ or $\left(\bar{p}_{w}, \bar{p}_{\tilde{w}}\right)=(0,1)$ (that is, $\left.\alpha \gg 1\right)$. Equating Eqs. 40 and 44, we obtain a formal expression for $\varepsilon$ in terms of the degree of asymmetry coefficient $\alpha$,

$$
\varepsilon(\alpha) \stackrel{\text { def }}{=} \alpha(1+\alpha)^{-2}
$$

From Eq. 45, we note that to get an $\varepsilon$ of the order of $10^{-2} \ll 1$, one needs to have $\alpha$ of the order of $10^{2}$. As previously mentioned, highly successful discrimination protocols characterized by very small minimum error probability values can be obtained when strong asymmetries between the prior probabilities $p_{w}$ and $p_{\tilde{w}}$ occur. In such cases, the values 
of $\delta_{\max }\left(\bar{p}_{w}\right)$ can be rather small. For instance, when $p_{w}=10^{3} p_{\tilde{w}}, \delta_{\max } \simeq 3.16 \times 10^{-2}$ and $\mathcal{F}_{\text {nearly-optimal }} \geq 0.999$. We point out that this order of magnitude of angles is quite small. Indeed, in real world settings one necessarily deals with imperfect measurements where intrinsic uncertainties yielding non-negligible systematic errors may be present. For instance, the intrinsic uncertainty of a polarization rotor, typically of the order of $2^{\circ}-4^{\circ}$ on the Bloch sphere (where $1^{\circ} \simeq 1.75 \times 10^{-2}$ rad.), limits the precision of the measurement on a polarization qubit [19].

For the sake of clarity, we point out that in our numerical calculations that appear into the next section, the two a priori probabilities $p_{w}$ and $p_{\tilde{w}}$ will not be arbitrarily chosen. They will be chosen in such a manner to yield highly successful discrimination protocols in which it is not difficult to discriminate between the exact and approximate quantum states despite their high degree of overlap. Such protocols are characterized by very low minimum error probability, which, in turn, is achievable when there is a sufficient degree of asymmetry $\alpha$ between the two a priori probabilities corresponding to the two states that we wish to distinguish (see Fig. 3). Then, to a larger $\alpha$ there corresponds a smaller $\varepsilon$. Specifically, we shall be considering asymmetries of the order of $10^{2}$ since they yield minimum error probability values of the order of $10^{-2}$ which are low enough for high degree of overlaps specified by angles of the order of $10^{-2} \mathrm{rad}$. Then, being in the framework of highly successful quantum discrimination protocols, we choose the accuracy the search has to achieve by imposing that the departure $\Delta \mathcal{F}$ of the unit fidelity $\mathcal{F}_{\text {optimal }}$ from the nearly

optimal desired fidelity value $\mathcal{F}_{\text {nearly-optimal }}$ must be smaller than the already small minimum error probability $p_{E}$. Imposing that $\delta_{\max }$ is of the order of the maximum achievable angular resolution in a typical quantum mechanical experiment [19], we find how asymmetric the two a priori probabilities must be chosen in order to guarantee the required accuracy the search should achieve.

In what follows, we compare the performances of the original and the modified Farhi-Gutmann analog quantum search algorithms. Clearly, both algorithms are stopped when they reach the same chosen lower bound.

\section{COMPARISON OF THE TWO SEARCH ALGORITHMS}

In this section, we identify a two-dimensional parametric region where the original algorithm is outperformed by the modified one provided one focuses on nearly optimal fidelity values compatible with the maximum resolution of the previously mentioned quantum discrimination protocol. For the sake of convenience, we shall refer to the original Farhi-Gutmann algorithm $(\gamma=1)$ as the special algorithm while the general algorithm denotes the modified version of the Farhi-Gutmann algorithm where $E^{\prime}=\gamma E$ with $\gamma \geq 1$. The transition probability $\mathcal{P}\left(t ; x, E, E^{\prime}\right)$ with $E^{\prime}=\gamma E$ 


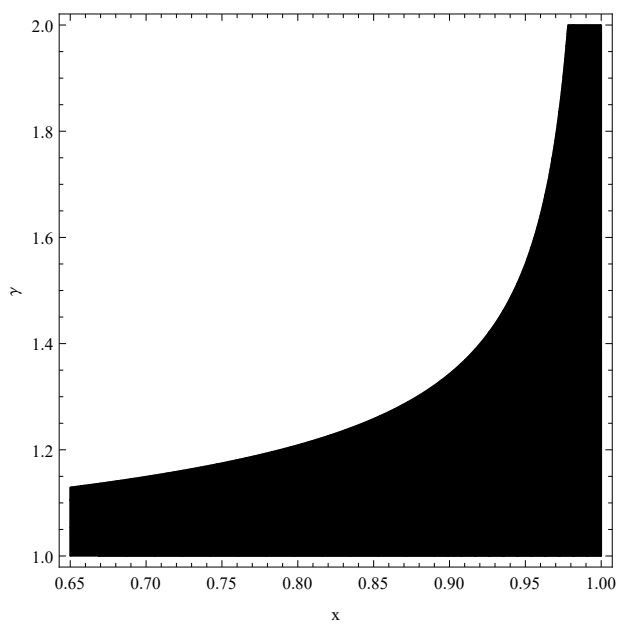

FIG. 4: Plot of the two-dimensional parametric region $r_{P}(x, \gamma)$ where the general algorithm outperforms the special algorithm in the working assumption that $p_{w}=10^{2} p_{\tilde{w}}$ and $P_{\text {threshold }}=99.5 \%$.

obtained for the general scenario in Eq. 32 is denoted as,

$$
\mathcal{P}_{\mathrm{g}}(t)=\frac{x^{2}(1+\gamma)^{2}}{4 x^{2} \gamma+(1-\gamma)^{2}} \sin ^{2}\left(\frac{E}{2 \hbar} \sqrt{4 x^{2} \gamma+(1-\gamma)^{2}} t\right)+x^{2} \cos ^{2}\left(\frac{E}{2 \hbar} \sqrt{4 x^{2} \gamma+(1-\gamma)^{2}} t\right)
$$

The maximum value of $\mathcal{P}_{\mathrm{g}}(t)$ in Eq. 46 is obtained at the time $\bar{t}_{\mathrm{g}}$,

$$
\bar{t}_{\mathrm{g}} \stackrel{\text { def }}{=} \frac{h}{4 E} \frac{2}{\sqrt{4 x^{2} \gamma+(1-\gamma)^{2}}},
$$

and, furthermore,

$$
\mathcal{P}_{\mathrm{g}}^{\max } \stackrel{\text { def }}{=} \mathcal{P}_{\mathrm{g}}\left(\bar{t}_{\mathrm{g}}\right)=\frac{x^{2}(1+\gamma)^{2}}{4 x^{2} \gamma+(1-\gamma)^{2}}
$$

Setting $\gamma=1$, we recover the main findings of Farhi and Gutmann. Specifically, the transition probability $\mathcal{P}_{\mathrm{g}}(t)$ in Eq. 46 reduces to $\mathcal{P}_{\mathrm{S}}(t) \stackrel{\text { def }}{=} \mathcal{P}_{\text {Farhi-Gutmann }}(t ; x, E)$ in Eq. 33. The maximum value of $\mathcal{P}_{\mathrm{s}}(t)$ is obtained at the time $\bar{t}_{\mathrm{s}}$,

$$
\bar{t}_{\mathrm{s}} \stackrel{\text { def }}{=} \frac{h}{4 E x},
$$

and, furthermore,

$$
\mathcal{P}_{\mathrm{s}}^{\max } \stackrel{\text { def }}{=} \mathcal{P}_{\mathrm{s}}\left(\bar{t}_{\mathrm{s}}\right)=1
$$

We propose to rank the performance of the two algorithms in two different ways given that Eq. 422 is satisfied: i) rank the two algorithms by comparing the two transitions probabilities evaluated at an identical fixed-value of the travel time; ii) rank the two algorithms by comparing the two minimum travel times needed to arrive at an identical 


\begin{tabular}{c|c|c|c|c|c|c}
\hline \hline$x$ & $\delta\left(\times 10^{-2}\right)$ & $P_{\mathrm{g}}^{\max }$ & $\Delta \mathcal{F}\left(\times 10^{-3}\right)$ & $p_{E}\left(\times 10^{-3}\right)$ & $t_{\mathrm{FG}}^{\text {(special) }}\left(\times 10^{-1}\right)$ & $t_{\mathrm{FG}}^{\text {(general) }}\left(\times 10^{-1}\right)$ \\
\hline \hline 0.65 & 5.56 & 0.9969 & 3.100 & 9.870 & 3.67 & 3.66 \\
\hline 0.70 & 4.85 & 0.9976 & 2.400 & 9.877 & 3.42 & 3.40 \\
\hline 0.75 & 4.20 & 0.9982 & 1.800 & 9.883 & 3.20 & 3.17 \\
\hline 0.80 & 3.57 & 0.9987 & 1.300 & 9.888 & 3.01 & 2.97 \\
\hline 0.85 & 2.95 & 0.9991 & 0.900 & 9.892 & 2.84 & 2.80 \\
\hline 0.90 & 2.31 & 0.9995 & 0.500 & 9.896 & 2.68 & 2.64 \\
\hline 0.95 & 1.56 & 0.9998 & 0.200 & 9.899 & 2.55 & 2.51 \\
\hline
\end{tabular}

TABLE I: Illustrative numerical estimates of $t_{\mathrm{FG}}^{\text {(special) }}$ and $t_{\mathrm{FG}}^{\text {(general) }}$ for given values of $P_{\mathrm{g}}^{\max }$ obtained by assuming $\gamma=1.1$ and $h=E=1$. For the selected values of the angle $\delta$, the numerical estimates of $p_{E}$ are computed by considering the relation $p_{\omega}=10^{2} p_{\tilde{\omega}}$ which yields $\delta_{\max } \simeq 9.92 \times 10^{-2}$.

fixed-value of the transition probability.In the first scenario, since $\bar{t}_{\mathrm{g}} \leq \bar{t}_{\mathrm{s}}$, we wish to determine whether or not there is, for a fixed value of $\bar{t}_{\mathrm{g}}$ less than $\bar{t}_{\mathrm{s}}$, a two-dimensional parametric region $\mathcal{R}_{t}(x, \gamma)$,

$$
\mathcal{R}_{t}(x, \gamma) \stackrel{\text { def }}{=}\left\{(x, \gamma) \in(0,1) \times[1, \infty): \mathcal{P}_{\mathrm{g}}^{\max }-\mathcal{P}_{\mathrm{s}}\left(\bar{t}_{\mathrm{g}}\right)>0\right\}
$$

where the general algorithm outperforms the special algorithm in terms of transition probability values in the working assumption of accepting transition probability values less than one.

In the second scenario, since $\mathcal{P}_{\mathrm{g}}^{\max } \leq \mathcal{P}_{\mathrm{s}}^{\max }$, we wish to determine whether or not there is, for a fixed value of $\mathcal{P}_{\mathrm{g}}^{\max }$ less than one, a two-dimensional parametric region $\mathcal{R}_{\mathcal{P}}(x, \gamma)$,

$$
\mathcal{R}_{\mathcal{P}}(x, \gamma) \stackrel{\text { def }}{=}\left\{(x, \gamma) \in(0,1) \times[1, \infty): \frac{\bar{t}_{\mathrm{g}}}{\tilde{t}_{\mathrm{s}}}<1\right\}
$$

where the general algorithm outperforms the special algorithm in terms of minimum travel times at fixed nearly optimal transition probability values.

Note that $\tilde{t}_{\mathrm{s}}$ in Eq. 52 is such that,

$$
\mathcal{P}_{\mathrm{s}}\left(\tilde{t}_{\mathrm{s}}\right)=\mathcal{P}_{\mathrm{g}}^{\max }
$$

where, after some algebraic manipulations, we find

$$
\tilde{t}_{\mathrm{s}} \stackrel{\text { def }}{=} \frac{h}{2 \pi E x} \cos ^{-1}\left[\left(\frac{1-\frac{x^{2}(1+\gamma)^{2}}{4 x^{2} \gamma+(1-\gamma)^{2}}}{1-x^{2}}\right)^{\frac{1}{2}}\right] .
$$


We emphasize that both two-dimensional parametric regions $\mathcal{R}_{t}(x, \gamma)$ and $\mathcal{R}_{\mathcal{P}}(x, \gamma)$ in Eqs. 51] and (52), respectively, are non-empty sets and in addition, it can be numerically verified that they are identical. Moreover, for the sake of completeness we also point out that one can investigate whether or not the general algorithm outperforms the special one for a given transition probability threshold $\mathcal{P}_{\text {threshold }}$ given that Eq. 42 is satisfied. For this reason, we consider the following sub-region $r_{\mathcal{P}}(x, \gamma)$ of $\mathcal{R}_{\mathcal{P}}(x, \gamma)$ in Eq. 52 ,

$$
r_{\mathcal{P}}(x, \gamma) \stackrel{\text { def }}{=}\left\{(x, \gamma) \in(0,1) \times[1, \infty): \frac{\bar{t}_{\mathrm{g}}}{\tilde{t}_{\mathrm{s}}}<1, \text { and } \mathcal{P}_{\mathrm{g}}^{\max }>\mathcal{P}_{\text {threshold }}\right\}
$$

A plot of the two-dimensional parametric region $r_{P}(x, \gamma)$ in Eq. 55 where the general algorithm outperforms the special algorithm when assuming $\mathcal{P}_{\text {threshold }}=99.5 \%$ and $p_{w}=10^{2} p_{\tilde{w}}$ appears in Fig. 4.

Finally, for the sake of clarity, we also present in Table I illustrative numerical estimates of $\bar{t}_{\mathrm{g}} \stackrel{\text { def }}{=} t_{\mathrm{FG}}^{\text {(general) }}$ in Eq. 47 and $\tilde{t}_{\mathrm{s}} \stackrel{\text { def }}{=} t_{\mathrm{FG}}^{\text {(special) }}$ in Eq. 54 for a number of selected values of the threshold probability $\mathcal{P}_{\mathrm{g}}^{\max }$ in Eq. 48 under the working assumptions that $\gamma=1.1$ and $h=E=1$. Given the chosen values of the angle $\delta$ in Table I, the numerical estimates of the minimum error probability $p_{E}$ are calculated by assuming $p_{w}=10^{2} p_{\tilde{w}}$ which yields a maximum value of $\delta$ that equals $\delta_{\max } \simeq 9.92 \times 10^{-2}$.

As a further clarifying remark, we point out that the numerical values (originated from our proposed comparison of the two algorithms and reported in Table I) were computed by considering the minimum time such that the desired success probability values were obtained. We have simply chosen the desired success probability values to be equal to the maximal success probability values of the modified algorithm in our analysis. However, as evident from Fig. 5, we could have chosen different threshold values (leading to shorter minimum times) and still outperform the original algorithm (provided that only a nearly optimal search is considered). As a side remark, we emphasize that this line of reasoning extends naturally to any desired success probability value taken as the maximal success probability $\mathcal{P}_{\text {threshold }}=\mathcal{P}_{\mathrm{g}}^{\max }$ with $\mathcal{P}_{\mathrm{g}}^{\max }=\mathcal{P}_{\mathrm{g}}^{\max }(x, \gamma)$ in Eq. 48 and where the points $\{(x, \gamma)\}$ can be chosen to belong to the black-colored region in Fig. 4. Specifically, in Fig. 5 we plot $\mathcal{P}_{g}(t)$ (dashed line) and $\mathcal{P}_{s}(t)$ (solid line) as a function of time $t$. For the sake of reasoning, we set $h=1, E=1, x=0.80$, and $\gamma=1.1$. The two horizontal lines in Fig. 5 denote two selected success probability values, $\mathcal{P}_{\text {threshold }}^{\max }=0.9987$ (dotted line) and $\mathcal{P}_{\text {threshold }}=0.9$ (dotted-dashed line). The threshold value 0.9987 was taken from the fourth line in Table I. We note that the minimum time such that the two desired success probability values are obtained is smaller in the case of the modified algorithm for both selected thresholds. Specifically, in the first and second scenarios, we have $1.93 \times 10^{-1} \leq 2.02 \times 10^{-1}$ and $2.97 \times 10^{-1} \leq 3.01 \times 10^{-1}$, respectively. Note that in the MKSA unit system, $[t]_{\mathrm{MKSA}}=[h]_{\mathrm{MKSA}}[E]_{\mathrm{MKSA}}^{-1}$. Since we have considered $E=1=h$ in our numerical computations, time $t$ is assumed to be dimensionless. 


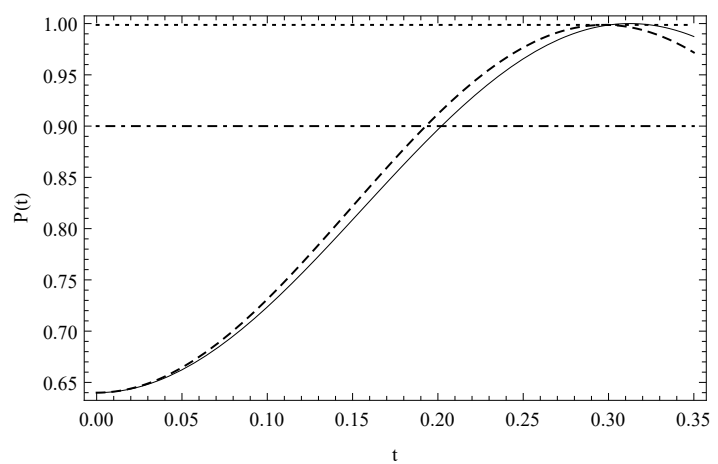

FIG. 5: Plot of $\mathcal{P}_{g}(t)$ (dashed line) and $\mathcal{P}_{s}(t)$ (solid line) versus $t$. We set $h=1, E=1, x=0.80$, and $\gamma=1.1$. The two horizontal lines denote two selected success probability values, $\mathcal{P}_{\text {threshold }}^{\max }=0.9987$ (dotted line) and $\mathcal{P}_{\text {threshold }}=0.9($ dot-dashed line). Observe that the minimum time such that the two desired success probability values are obtained is smaller in the case of the modified algorithm for both selected thresholds. Specifically, in the first and second scenarios, we have $1.93 \times 10^{-1}$ $\leq 2.02 \times 10^{-1}$ and $2.97 \times 10^{-1} \leq 3.01 \times 10^{-1}$, respectively

Finally, for a more detailed discussion on the probability of small and large numerical values of the quantum overlap $x$, we refer to Appendix A.

\section{CONCLUDING REMARKS}

In this paper, we focused on the problem of nearly optimal state searching and we demonstrated that it is possible to modify the original Farhi-Gutmann search Hamiltonian (see Eq. (28)) in order to speed up the procedure for finding a suitably distributed unknown normalized quantum state $|w\rangle$ under the working assumption that only a nearly optimal fidelity is achieved,

$$
\mathcal{P}_{\max }(\delta) \stackrel{\text { def }}{=} \cos ^{2}(\delta)
$$

with $0 \leq \delta \stackrel{\text { def }}{=} \cos ^{-1}[\langle w \mid \tilde{w}\rangle] \ll 1$ where $|w\rangle$ and $|\tilde{w}\rangle$ denote the exact and the approximate target states, respectively. More specifically, upon relaxing the working assumptions of exact state overlap and uniform distribution of the target state on the unit sphere in the $N$-dimensional complex Hilbert space, we showed that the proposed algorithm (see Eq. (28) ) can indeed outperform the original analog counterpart of a quantum search algorithm. This enhanced performance occurs for a convenient choice of both the ratio $\gamma \stackrel{\text { def }}{=} E^{\prime} / E$ between the eigenvalues of the modified search Hamiltonian and the quantum mechanical overlap $x \stackrel{\text { def }}{=}\langle w \mid s\rangle$ between the initial $(|s\rangle)$ and the target $(|w\rangle)$ states.

In summary, the main results of our work can be described as follows. First, we extended the optimality proof 
presented in Ref. [3] to the case of nearly optimal state searching and determined that the minimum time interval to find the target state with probability $\mathcal{P}_{\max }(\delta) \stackrel{\text { def }}{=} \cos ^{2}(\delta)$ is given by (see Eq. 27.),

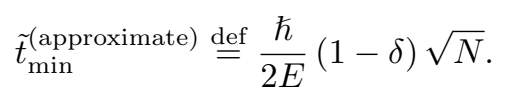

Second, we computed the transition probability $\mathcal{P}\left(t ; x, E, E^{\prime}\right)$ (see Eq. 32 ) that arises from our proposed search algorithm and determined its maximum value $\mathcal{P}_{\max }(x, \gamma)$ (see Eq. 34) and Fig. 1) together with the instant $t^{*}(x, \gamma)$ (see Eq. (36) ) at which this maximum is achieved. Third, from the formal introduction of the quantity $\delta$ in Eq. (3) and the expression of $\mathcal{P}_{\max }(x, \gamma)$ in Eq. 34), we found an explicit expression for the quantity $\delta=\delta(x, \gamma)$ (see Eq. (38) and Fig. 2) responsible for our imperfect state search in terms of the two parameters $x$ and $\gamma$ that characterize our search Hamiltonian,

$$
\delta(x, \gamma) \stackrel{\text { def }}{=} \cos ^{-1}\left[\frac{(1+\gamma) x}{\sqrt{(1-\gamma)^{2}+4 \gamma x^{2}}}\right] .
$$

Fourth, we introduced the notion of approximate target state $|\tilde{w}\rangle$. Such a quantum state $|\tilde{w}\rangle$ is characterized by a sufficiently large overlap with the exact target state $|w\rangle$ so that, as we proposed, the deviation $\Delta \mathcal{F}(\delta)$ from the unit fidelity $\mathcal{F}=1$ is smaller than the minimum error probability $p_{E}(\delta)$ achievable in an optimal quantum state discrimination protocol (see Fig. 3) for the set of quantum states $\{|w\rangle,|\tilde{w}\rangle\}$,

$$
0 \leq \Delta \mathcal{F}(\delta)=1-\cos ^{2}(\delta) \leq p_{E}(\delta)
$$

with $p_{E}(\delta)$ defined in Eq. 41]. Finally, using numerical methods, we showed that for a fixed transition probability threshold $0 \leq \mathcal{P}_{\text {threshold }}<1$ and suitably chosen non-uniform probability distribution functions of the target state on the unit sphere (see Eq. (A4)), there exist two-dimensional parametric regions $r_{P}(x, \gamma)$ (see Eq. (55)) in which the proposed algorithm outperforms the original one in terms of speed (see Fig. 4, Table I, and Fig. 5) for the special task (namely, finding the approximate target state) it sets out to solve.

We believe that our analysis presented in this paper can serve as a relevant starting point for a more rigorous investigation that would include both theoretical and experimental aspects of the tradeoff between fidelity and run time of quantum search algorithms. For instance, it is known that to mitigate the effect of decoherence originating from the interaction of a quantum system with the environment, it is helpful to decrease the control time of the control fields employed to generate a target quantum state or a target quantum gate. Simultaneously, to enhance the fidelity of generating such targets and reach values arbitrarily close to the maximum $\mathcal{F}=1$, it may be convenient to increase the control time beyond a certain critical value. When the control time reaches a certain value that may be 
close to the just mentioned critical value however, decoherence can become a dominant effect. Therefore, investigating the tradeoff between fidelity and time control can be of great practical importance in quantum computing 30 . For instance, one can design algorithms seeking suboptimal control solutions for much reduced computational effort, since it is very challenging to find a rigorous optimal time control and in many cases the control is only required to be sufficiently precise and short. For example, the fidelity of tomography experiments is rarely above $99 \%$ due to the limited control precision of the tomographic experimental techniques as pointed out in Ref. [31]. Under such conditions, it is unnecessary to prolong the control time since the departure from the optimal scenario is essentially negligible. Hence, it can certainly prove worthwhile to design slightly suboptimal algorithms that can be much cheaper computationally. Our analysis can be improved in a number of ways. First, our work can be strengthened by explicitly considering the quantum measurement process in our analysis. For instance, it is known that quantum measurement can be regarded as a decoherence process that potentially enhance or reduce a transition probability between two quantum states [16. Second, as a starting point of our investigation, we could consider a more general time-independent search Hamiltonian where the probability of obtaining the target state can be very large and nearly one 33]. Furthermore, in order to work in a more realistic setting, nearly optimal time-dependent search Hamiltonians specified in terms of the so-called schedule function of the search algorithm could also be considered [34. Finally, from a more ambitious perspective, we could explore the manner in which recent investigations on intelligent forms of quantum searching algorithms in the presence of imperfections, with the help of techniques borrowed from quantum machine learning [35, would connect to our nearly optimal quantum search problem.

In conclusion, we hope to pursue a more rigorous and realistic analysis that includes these intriguing theoretical and experimental aspects in forthcoming scientific efforts.

\section{Acknowledgments}

C. C. is grateful to the United States Air Force Research Laboratory (AFRL) Summer Faculty Fellowship Program for providing support for this work. Any opinions, findings and conclusions or recommendations expressed in this paper are those of the authors and do not necessarily reflect the views of AFRL. Finally, constructive criticism from two anonymous referees leading to an improved version of this manuscript are sincerely acknowledged by the authors.

[1] L. K. Grover, Quantum mechanics helps in searching for a needle in a haystack, Phys. Rev. Lett. 79, 325 (1997). 
[2] D. E. Knuth, The Art of Computer Programming, Vol. 3: Sorting and Searching, Addison-Wesley, Reading, MA (1975).

[3] E. Farhi and S. Gutmann, Analog analogue of a digital quantum computation, Phys. Rev. A57, 2403 (1998).

[4] C. Cafaro and S. Mancini, An information geometric viewpoint of algorithms in quantum computing, in Bayesian Inference and Maximum Entropy Methods in Science and Engineering, AIP Conf. Proc. 1443, 374 (2012).

[5] C. Cafaro and S. Mancini, On Grover's search algorithm from a quantum information geometry viewpoint, Physica A391, $1610(2012)$.

[6] C. Cafaro, Geometric algebra and information geometry for quantum computational software, Physica A470, 154 (2017).

[7] L. Mandelstam and I. Tamm, The uncertainty relation between energy and time in non-relativistic quantum mechanics, J. Phys. USSR 9, 249 (1945).

[8] L. Vaidman, Minimum time for the evolution to an orthogonal quantum state, Am. J. Phys. 60, 182 (1992).

[9] J. Uffink, The rate of evolution of a quantum state, Am. J. Phys. 61, 935 (1993).

[10] N. Margolus and L. B. Levitin, The maximum speed of dynamical evolution, Physica D120, 188 (1998).

[11] L. B. Levitin and T. Toffoli, Fundamental limit on the rate of quantum dynamics: The unified bound is tight, Phys. Rev. Lett. 103, 160502 (2009).

[12] J. Soderholm, G. Bjork, T. Tsegaye, and A. Trifonov, States that minimize the evolution time to become an orthogonal state, Phys. Rev. A59, 1788 (1999).

[13] V. Giovannetti, S. Lloyd, and L. Maccone, Quantum limits to dynamical evolution, Phys. Rev. A67, 052109 (2003).

[14] M. H. Partovi, Entropic formulation of uncertainty for quantum measurements, Phys. Rev. Lett. 50, 1883 (1983).

[15] H. F. Hofmann, T. Kobayashi, and A. Furusawa, Correlations of measurement information and noise in quantum measurements with finite resolution, arXiv:quant-ph/0003067 (2000).

[16] T. Fritz, Transition probabilities and measurement statistics of postselected ensembles, J. Math. Phys. 51, 082105 (2010).

[17] A. Chefles, Quantum state discrimination, Contemporary Physics 41, 401 (2000).

[18] S. M. Barnett and S. Croke, Quantum state discrimination, Advances in Optics and Photonics 1, 238 (2009).

[19] D. Rosset, R. Ferretti-Schöbitz, J.-D. Bancal, N. Gisin, and Y.-C. Liang, Imperfect measurement settings: Implication for quantum state tomography and entanglement witnesses, Phys. Rev. A86, 062325 (2012).

[20] M. T. Di Mario and F. E. Becerra, Robust measurement for the discrimination of binary coherent states, Phys. Rev. Lett. 121, 023603 (2018).

[21] I. D. Ivanovic, How to differentiate between non-orthogonal states, Phys. Lett. A123, 257 (1987).

[22] D. Dieks, Overlap and distinguishability of quantum states, Phys. Lett. A126, 303 (1988).

[23] A. Peres, How to differentiate between non-orthogonal states, Phys. Lett. A128, 19 (1988).

[24] A. S. Holevo, Statistical decision theory for quantum systems, J. Multivariate Analysis 3, 377 (1973).

[25] H. P. Yuen, R. S. Kennedy, and M. Lax, Optimum testing of multiple hypotheses in quantum detection theory, IEEE Trans. 
Inf. Theory IT-21, 125 (1975).

[26] C. W. Helstrom, Quantum Detection and Estimation Theory, Academic Press, New York (1976).

[27] A. M. Childs, J. Preskill, and J. Renes, Quantum information and precision measurement, J. Mod. Optics 47, 155 (2000).

[28] C. A. Fuchs and J. van de Graaf, Cryptographic distinguishability measures for quantum-mechanical states, IEEE Trans. Inf. Theor. 45, 1216 (1999).

[29] K. M. R. Audenaert, Comparisons between quantum state distinguishability measures, Quantum Information and Computation 14, $31(2014)$.

[30] K. W. Moore Tibbetts, C. Brif, M. D. Grace, A. Donovan, D. L. Hocker, T.-S. Ho, R.-B. Wu, and H. Rabitz, Exploring the tradeoff between fidelity and time optimal control of quantum unitary transformations, Phys. Rev. A86, 062309 (2012).

[31] Q.-M. Chen, R.-B. Wu, T.-M. Zhang, and H. Rabitz, Near-time-optimal control for quantum systems, Phys. Rev. A92, $063415(2015)$.

[32] M. Hirose and P. Cappellaro, Time-optimal control with finite bandwidth, Quantum Information Processing 17, 88 (2018).

[33] J. Bae and Y. Kwon, Generalized quantum search Hamiltonian, Phys. Rev. A66, 012314 (2002).

[34] A. M. Dalzell, T. J. Yoder, and I. L. Chuang, Fixed-point adiabatic quantum search, Phys. Rev. A95, 012311 (2017).

[35] J. Biamonte, P. Wittek, N. Pancotti, P. Rebentrost, N. Wiebe, and S. Lloyd, Quantum machine learning, Nature 549, 195 $(2017)$.

[36] L. Ghirardi, I. Siloi, P. Bordone, F. Troiani, and M. G. A. Paris, Quantum metrology at level anticrossing, Phys. Rev. A97, $012120(2018)$

[37] K. Bartkiewicz and A. Miranowicz, Optimal cloning of qubits given by an arbitrary axisymmetric distribution on the Bloch sphere, Phys. Rev. A82, 042330 (2010).

[38] P. Kang, H.-Y. Dai, J.-H. Wei, and M. Zhang, Optimal quantum cloning based on the maximin principle by using a priori information, Phys. Rev. A94, 042304 (2016).

[39] C. Cafaro and S. A. Ali, Maximum caliber inference and the stochastic Ising model, Phys. Rev. E94, 052145 (2016).

[40] A. S. Fletcher, P. Shor, and M. Z. Win, Channel-adapted quantum error correction for the amplitude damping channel, IEEE Trans. Inf. Theor. 54, 5705 (2008).

[41] C. Cafaro and P. van Loock, Approximate quantum error correction for generalized amplitude-damping errors, Phys. Rev. A89, 022316 (2014).

[42] C. Cafaro and P. van Loock, A simple comparative analysis of exact and approximate quantum error correction, Open Systems \& Information Dynamics 21, 1450002 (2014).

[43] M. Tiersch, E. J. Ganahl, and H. J. Briegel, Adaptive quantum computation in changing environments using projective simulation, Scientific Reports 5, 12874 (2015).

[44] V. Dunjko, J. M. Taylor, and H. J. Briegel, Quantum-enhanced machine learning, Phys. Rev. Lett. 117, 130501 (2016). 


\section{Appendix A: Numerical values of the quantum overlap}

In this Appendix, we present a more in depth discussion on the probability of small and large numerical values of the quantum overlap $x$.

In general, one may argue that large values of the overlap $x$ are not very typical or particularly realistic since they occur when the source state is essentially already the desired target state. This objection has its merit when one assumes that the realistic scenario is the one in which no a priori information on the target state is available and, in particular, the target state is assumed to be a normalized vector in a list of $N$ possible orthonormal states. Indeed, within these working assumptions, $x=N^{-\frac{1}{2}}$ and the largest value of $x$ that would be considered realistic is obtained for $N=4$ and equals $x=1 / 2$. However, one can envision a circumstance in which a priori relevant knowledge is available so that the target state is a suitably chosen non-uniformly distributed normalized vector in a $N$-dimensional vector space. In such a scenario, the probability of occurrence of large values of $x$ can begin to be non-negligible. Thus, large values of $x$ can become realistic in these new working conditions. We point out that $a$ priori knowledge happens to be useful in both quantum metrology and imperfect (optimal) quantum cloning. In the former case, in order to design the best parameter estimation scheme, some form of a priori knowledge of such a parameter is required 36. In the latter case, instead, knowledge of the distribution of qubits on the Bloch sphere that encodes some a priori information on a given quantum state that one desires to clone is taken advantage of in order to achieve the highest fidelity [37, 38. For instance, in the specific framework of phase-independent quantum cloning, one assumes to clone qubits that are a priori known to be symmetrically distributed around the Bloch vector [37].

As pointed out in Section II, the optimality proof by Farhi and Gutmann occurs under the special working assumption that the target state $|w\rangle$ is an unknown element of a given orthonormal basis $\{|a\rangle\}$ with $1 \leq a \leq N$ of an $N$-dimensional complex Hilbert space $\mathcal{H}_{2}^{n}$ with $N \stackrel{\text { def }}{=} 2^{n}$. As pointed out earlier, in this scenario it is rather unlikely

that $x=N^{-\frac{1}{2}}$ assumes values close to one. In their more general setting however, Farhi and Gutmann assume that the target state $|w\rangle$ is an arbitrary normalized vector in a $N$-dimensional complex Hilbert space which is uniformly distributed on the unit sphere [3].

We briefly recall that the space enclosed by a $(2 N-1)$-dimensional unit sphere $\mathcal{S}^{2 N-1}$ is a $(2 N)$-ball whose infinitesimal volume element in spherical coordinates is given by,

$$
d V_{2 N \text {-ball }}^{\text {(spherical) }} \stackrel{\text { def }}{=} r^{2 N-1} \sin ^{2 N-2}\left(\theta_{1}\right) \sin ^{2 N-3}\left(\theta_{2}\right) \ldots \sin \left(\theta_{2 N-2}\right) d r d \theta_{1} d \theta_{2} \ldots d \theta_{2 N-2} d \theta_{2 N-1}
$$

where $\theta_{i} \in[0, \pi)$ for any $1 \leq i \leq 2 N-2$ and $\theta_{2 N-1} \in[0,2 \pi)$. Furthermore, the volume element of the $(2 N-1)$ - 
dimensional unit sphere generalizes the concept of the area element of a two-dimensional unit sphere and, from Eq. A1, is given by

$$
d V_{\mathcal{S}^{2 N-1}}^{\text {(spherical) }} \stackrel{\text { def }}{=} \sin ^{2 N-2}\left(\theta_{1}\right) \sin ^{2 N-3}\left(\theta_{2}\right) \ldots \sin \left(\theta_{2 N-2}\right) d r d \theta_{1} d \theta_{2} \ldots d \theta_{2 N-2} d \theta_{2 N-1}
$$

Returning to our discussion, we assume that the probability density function $\rho_{w}(\theta)$ that describes the distribution of the states $|w\rangle$ on the $(2 N-1)$-dimensional unit sphere depends on the coordinate $\theta_{1}$, denoted as $\theta$, which is uniform with respect to the remaining coordinates. Therefore, by marginalizing over all the unimportant integration variables but $\theta$ with $x \stackrel{\text { def }}{=}|\langle s \mid w\rangle|=\cos (\theta)$ where $0 \leq \theta \leq \pi / 2$, we find that the probability that $x$ is greater than a given value $\bar{x}$ is given by $[3$,

$$
\operatorname{Prob}(x \geq \bar{x}) \stackrel{\text { def }}{=} \frac{\int_{0}^{\cos ^{-1}(\bar{x})} \rho_{w}(\theta)[\sin (\theta)]^{2 N-2} d \theta}{\int_{0}^{\frac{\pi}{2}} \rho_{w}(\theta)[\sin (\theta)]^{2 N-2} d \theta}
$$

The quantity $\rho_{w}(\theta)$ in Eq. A3 denotes a well-defined probability density function (pdf), that is to say, a pdf that is positive and normalized to one. For the sake of reasoning, we select $|s\rangle$ to be at the north pole. Generalizations to less peculiar scenarios are straightforward. In what follows, we provide some rationale for our choice of $\rho_{w}(\theta)$. The functional form of $\rho_{w}(\theta)$ is essentially that of a Gaussian with mean $\mu_{\theta}$ and variance $\sigma_{\theta}^{2}$ multiplied by a suitably chosen oscillatory function. The mean is set equal zero, while any value of $\mu_{\theta}$ between 0 and $\pi / 2$ can be chosen provided that the variance $\sigma_{\theta}^{2}$ is not too small. Furthermore, the multiplying factor in the proposed expression of $\rho_{w}(\theta)$ is chosen in such a manner as to substantially mitigate the oscillatory behavior of $[\sin (\theta)]^{2 N-2}$ in Eq. A3 and leads when multiplied with it, to an approximately constant function over the selected domain of integration. Practically, one can consider a narrowly distributed Gaussian peaked nearby the location of the initial state or, for a Gaussian peaked far away from such a location, the width of the Gaussian has to be suitably larger. Under this assumption, a convenient choice for our analysis is given by the following pdf,

$$
\rho_{w}(\theta) \stackrel{\text { def }}{=} \mathcal{N} \frac{\exp \left(-\frac{\theta^{2}}{2 \sigma_{\theta}^{2}}\right)}{1+[10 \sin (\theta)]^{2 N-2}} .
$$

In Eq. A4, $\mathcal{N}=\mathcal{N}\left(N, \sigma_{\theta}^{2}\right)$ is a normalization factor that depends on the choice of $N$ and $\sigma_{\theta}^{2}$. For instance, for $N=16, \sigma_{\theta}^{2}=1$, and $\bar{x}=0.95$, by means of numerical integration, we find $\operatorname{Prob}(x \geq 0.95) \simeq 21 \%$. We note that with a smaller $\sigma_{\theta}^{2}$, the probability of $x$ being greater than a selected value $\bar{x}$ increases and asymptotically approaches unity. For instance, for $\sigma_{\theta}^{2}=10^{-1}$ and $\sigma_{\theta}^{2}=10^{-2}$, we observe that $\operatorname{Prob}(x \geq 0.95) \simeq 58 \%$ and $\operatorname{Prob}(x \geq 0.95) \simeq 99 \%$, respectively. We point out that if $N=16$ and $\rho_{w}(\theta)$ is uniform as selected in Ref. [3], $\operatorname{Prob}(x \geq 0.95) \simeq 3.2 \times 10^{-17}$. Therefore, although the probability is not exactly zero, it is very unlikely that $x$ is close to 1 when assuming uniformity 
for $\rho_{w}(\theta)$. For this reason, we considered here nearly optimal (imperfect) state searches where the target state is not uniformly distributed on the unit sphere. As a final remark, we point out that the assumption that the target state $|w\rangle$ is selected at random means that we assume absolute ignorance (that is, maximum entropy) about the location of the target. If we somehow learn an important piece of information about the location of the target however, one can think of updating his/her state of knowledge (see Ref. [39, for instance) about the target with a new probability density function of the state on the $(2 N-1)$-dimensional space. As a consequence, the search algorithm can be adapted to the target in order to improve the efficiency of the searching scheme. We emphasize that these considerations are reminiscent of what happens in channel-adapted quantum error correction [40 42] and adaptive quantum computing [43] where classical learning techniques can be used to enhance the performance of certain quantum tasks [44]. We leave the exploration of these intriguing ideas to future investigations. 